\title{
Efeitos da fotobioestimulação e terapia fotodinâmica antimicrobiana associadas a enxertos para recobrimento radicular de recessões gengivais múltiplas: ensaio Clínico randomizado controlado
}

\section{RESUMO}

As recessões gengivais (RG) múltiplas podem ser desafiadoras para o recobrimento radicular (RecRad). Tratamentos adjuvantes podem beneficiar os desfechos clínicos e centrados no paciente. O objetivo desse estudo é avaliar o potencial de duas terapias adjuvantes por laser no RecRad de RG múltiplas. E também, avaliar ferramentas tridimensionais de mensuração da cor gengival pelo sistema CIE L*a* $b^{*}$. Pacientes ( $\mathrm{n}$ : 54) com RGs múltiplas maxilares foram submetidos ao tratamento (TRAT) padronizado por raspagem da superfície radicular, enxerto de tecido conjuntivo subepitelial ( $1,0 \mathrm{~mm}$ de espessura) seguido do tracionamento coronal do retalho. Os grupos experimentais foram: grupo terapia fotodinâmica antimicrobiana (TFD) (n: 18) além do TRAT recebeu aplicação sob a porção radicular exposta de corante azul de toluidina $\mathrm{O}$ ( $\mathrm{pH}$ 3-4/1minuto) e aplicação de laser InAIGaP 660nm;

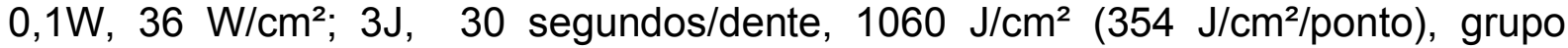
fotobioestimulação (FBM) (n: 18) após o TRAT sob retalho recebeu aplicação laser InAIGaP 660nm; 0,1W, $36 \mathrm{~W} / \mathrm{cm}^{2} ; 5 \mathrm{~J}, 50$ segundos/dente, $1770 \mathrm{~J} / \mathrm{cm}^{2}$ (354 $\mathrm{J} / \mathrm{cm}^{2} /$ ponto) e grupo controle ( $\left.\mathrm{n}: 18\right)$ TRAT + sham. Os pacientes foram acompanhados até 6 meses após o procedimento. Foram coletados dados clínicos, desfechos centrados nos pacientes e questionários de qualidade de vida (OHIP 14 e OIDP). Fotografias foram realizadas em todos os períodos de pós operatório, além da mensuração da cor gengival a partir de um espectrofotômetro de reflectância ajustado para conferir resultados no eixo $L^{*}, a^{*}, b^{*}$. Foi avaliada a correlação entre a variação de cor $(\Delta \mathrm{E})$ e espessura gengival pelo coeficiente de correlação de Spearman. Também foram avaliados o grau de acordo entre as medidas e comparação entre métodos de mensuração da cor gengival por espectrofotômetro e fotografias com auxílio de software. Todos dados foram avaliados estatisticamente tomando como referência o valor de $(p<0,05)$. Aos três e seis meses o grupo FBM apresentou os melhores resultados no completo RecRad $(p<0,05)$, porém sem diferença em relação aos outros grupos nos demais desfechos clínicos. A média de RecRad foi C: $81 \% \pm$ $17 \%$, TFD: $82,5 \% \pm 23 \%$ e FBM: $89 \% \pm 14 \%$ ( $p>0,05)$. Houve melhores resultados para o índice de cicatrizes, integração estética e cor, além dos desfechos relacionados no paciente: estética geral, redução da hipersensibilidade dentinária e redução do impacto na qualidade de vida para o grupo FBM em algum período de pós operatório em relação aos grupos C e TFD $(p<0,05)$. Já os grupos $C$ e TFD apresentaram variações $(p<0,05)$ em relação ao período inicial, mas sem diferença entre eles $(p>0,05)$ nesses parâmetros. A variação da espessura gengival não interferiu na mensuração da cor pelo espectrofotômetro, que apresentou maior grau de acordo entre diferentes medidas realizadas e, portanto, maior confiabilidade em relação ao método fotográfico de avaliação da cor. Apesar das limitações do estudo é possível concluir que o grupo FBM apresentou melhores resultados para o completo RecRad nessa amostra, porém na maioria dos parâmetros clínicos, não apresentou efeito adicional em relação aos grupos TFD e C. E o método de avaliação da cor gengival por espectrofotômetro demonstrou maior assertividade, não sofrendo alterações na mensuração quando ocorrem variações na espessura gengival.

Palavras-chave: Recessão gengival; Estética; Hiperestesia; Periodontia. 